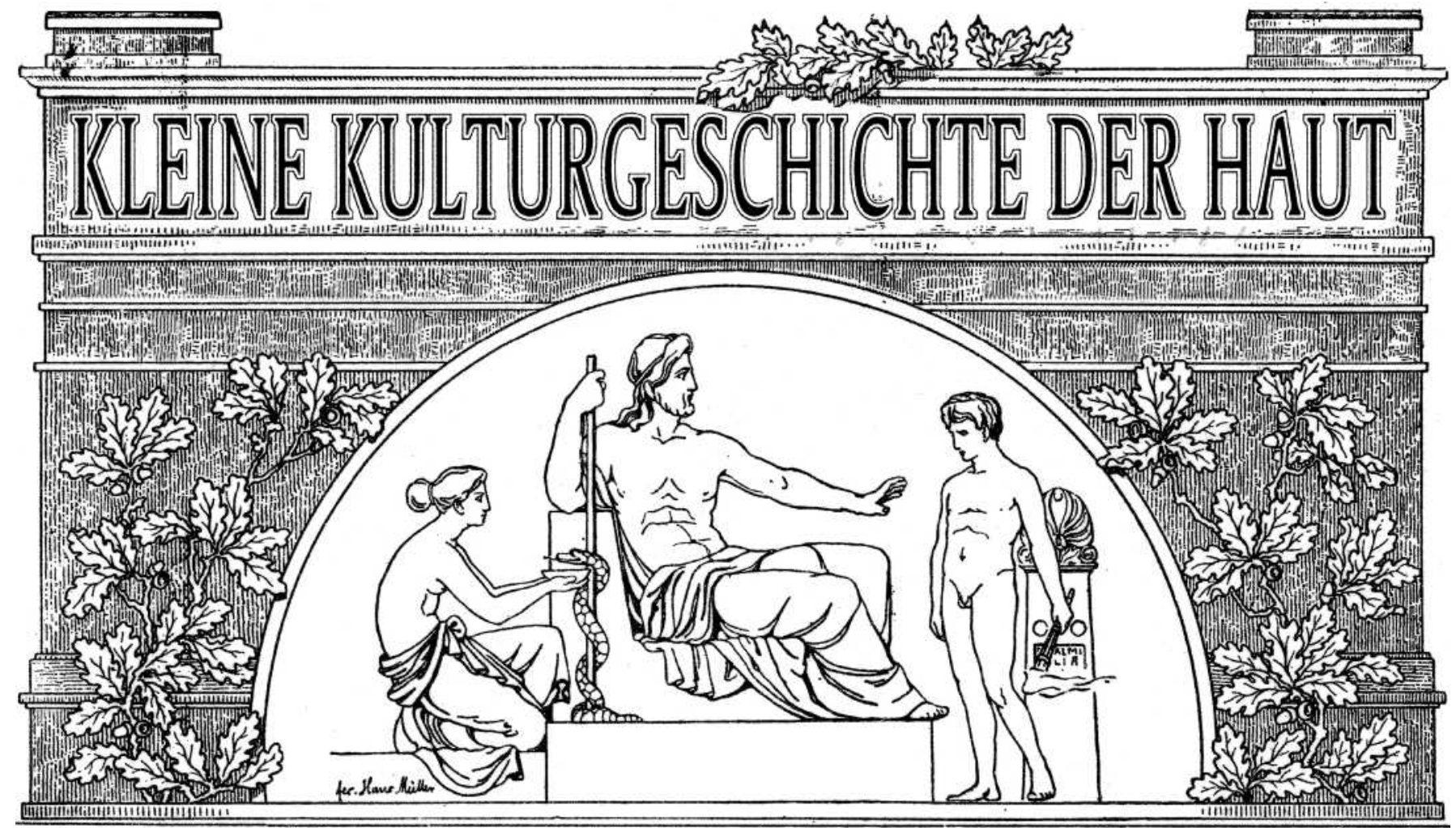

Die Zeitschrift „Aktuelle Dermatologie“ öffnet diese Rubrik und wird in loser Folge kurze, prägnante und informative Artikel darbringen zu vielen Aspekten der Kulturgeschichte in Beziehung zur Haut. Dies erstreckt sich auf alle Kulturen und Zeiten, auf die Künste, auf Mythen und Psychologie sowie natürlich auch auf medizinische Aspekte. Wir hoffen, dem geneigten Leser dadurch Interesse zu wecken, den Blick $z u$ weiten und Freude zu bereiten. Wir wünschen dieser Rubrik eine gute Aufnahme bei unseren Lesern und sind dankbar für Ihre Kommentare und Vorschläge für eine kleine Kulturgeschichte der Haut.

\title{
Weiße Indianer in Nordamerika
}

\section{Reitz}

Die genaue Herkunft der ersten Amerikaner liegt immer noch in einem rätselhaften Dunkel. Es gab Hauptwanderwellen aus Asien, aber auch bisher ungeklärte kleinere Einwanderungen aus anderen Kontinenten. Gesichert ist allein, dass die große Mehrheit der Vorfahren der amerikanischen Urbevölkerung einst in mindestens drei großen Wellen über eine Landbrücke zwischen Sibirien und Alaska in die Neue Welt eingewandert ist. Diese Landbrücke glich einem kleinen Kontinent, war einige tausend Kilometer breit und wurde Beringia genannt. Sie bildete sich während der Eiszeit in den vergangenen 100000 Jahren mindestens zweimal aus und war nicht vereist. Vor rund 50000 bis 40000 Jahren sowie vor rund 25000 bis 14000 Jahren waren so gewaltige Wassermassen im Eis gebunden, dass der Meeresspiegel um etwa 100 Meter niedriger lag als in der Gegenwart. Damit waren auch die Küstenlinien völlig verändert und große Teile der Landmassen waren leichter zu erreichen als nach dem Ende der Eiszeit.
Vermutlich kam der moderne Mensch, Homo sapiens, während seiner Ausbreitung über die Erde erst vor rund 30000 Jahren in Sibirien an, so dass Beringia von ihm nur während einer der letzten Trockenphasen durchschritten werden konnte. Tatsächlich belegen bei Indianern vergleichende Analysen des männlichen Y-Chromosoms, dass seit etwa 18000 Jahren in Nordamerika Menschen leben. Das Y-Chromosom kann zu 95\% mit dem X-Chromosom keine Rekombination eingehen, so dass es über die Generationen hinweg unverändert bleibt und aufgrund von regelmäßigen spontanen Veränderungen einem molekularen Kalender gleicht. Außerdem wurden sowohl in Nord- als auch in Südamerika noch nie menschliche Fossilien gefunden, die älter als 13500 Jahre sind. Indirekte Hinweise sprechen allerdings dafür, dass es möglicherweise bereits vor rund 30000 Jahren in Amerika Menschen gegeben haben könnte [1,2].

\section{Der Kennewick-Mann}

Asien gilt als der Ursprungsort der modernen menschlichen Großrassenentwicklung. Neben den Mongoloiden haben sich dort wahrscheinlich auch die Vorformen der Europiden und Negriden 
gebildet. Später wanderten diese Vorformen aus. Die frühen Präeuropiden zog es nach dem Westen, wo sie in Europa die Cro-Magnon-Menschen verdrängten. Die Ainu in Nordjapan verfügen noch heute über zahlreiche präeuropide Merkmale und gelten als Reste einer Mischbevölkerung zwischen den Prämongoloiden und den Präeuropiden. Da die Ainu eine hellere Haut als die typischen Mongoloiden besitzen, kann angenommen werden, dass auch die frühen Bewohner Sibiriens relativ hellhäutig waren. Insgesamt könnten die Menschen der ersten Wanderwellen nach Amerika sowohl von einer prämongoloiden als auch von einer präeuropiden Herkunft gewesen sein [3].

Im Jahre 1996 wurden am Ufer des Flusses Columbia in Benton County im US-Staat Washington Teile eines freigespülten menschlichen Skeletts gefunden, das zunächst für einen neuzeitlichen Europiden gehalten wurde. Die Untersuchung des Skelettes verwies auf einen Mann im Alter zwischen 40 und 45 Jahren und einer Größe von 1,73 Metern. Misstrauisch wurden die Untersucher durch eine uralte Pfeilspitze in der Hüfte des Mannes. Sie ließen deshalb das Alter des Skelettes bestimmen und kamen auf 9330 bis 9380 Jahre. Später wurde der Tote als Kennewick-Mann bezeichnet $[1,4]$. Er ist einer der ältesten bekannten Amerikaner. Das nach dem Schädel rekonstruierte Gesicht des Mannes belegte, dass er nicht wie ein typischer Indianer aussah (Abb.1).

\section{Die Rätsel der Clovis-Kultur}

In einer größeren Zahl konnten Menschen erst in Nordamerika einwandern, als sich auf dem Gebiet des heutigen Alaska und Kanada in den riesigen Gletschermassen eisfreie Korridore öffneten. Vorher war es nicht möglich, sich bei dem langen Weg über die Eisflächen durch Jagd sicher zu ernähren. Nur vereinzelt wagten sich während dieser Frühzeit Menschen entlang der Küstenlinien mit kleinen Booten von Sibirien nach Amerika. Ein erster eisfreier Korridor öffnete sich vor rund 14000 Jahren. Ihn nutzten die asiatischen Einwanderer, die in einer der ersten großen Siedlungswellen vor etwa 11000 bis 12000 Jahren auf dem Gebiet der heutigen USA die so genannte Clovis-Kultur gründeten; die älteste in Nordamerika sicher nachgewiesene menschliche Kultur. Die Hinterlassenschaften der Clovis-Menschen sind allerdings voller Rätsel. Ihre steinernen Pfeil- und Speerspitzen stimmen im Aussehen und der Herstellungstechnik nicht mit vergleichbaren Spitzen der damaligen Bewohner von Sibirien überein, sondern verweisen nach Europa. Die Clovis-Menschen produzierten ihre Spitzen nach der gleichen Technik wie die Bewohner des Solutréen im heutigen Frankreich und Spanien. Solutréen-Spitzen wurden in Europa vor 22000 bis 16500 Jahren hergestellt und gelten unter allen weltweiten Funden als die besten Pfeil- und Speerspitzen der damaligen Zeit. Es ist wahrscheinlich, dass die Clovis-Menschen die Herstellungstechniken von den europäischen Solutréen-Menschen gelernt haben. Sie mußten deshalb in Nordamerika Menschen aus Europa begegnet sein $[1,4]$.

Für die Wanderung der europäischen Solutréen-Menschen nach Amerika sprechen auch genetische Untersuchungen. Bei der Analyse der mitochondrialen DNA von nordamerikanischen Indianern können die Haplogruppen A bis D sowie insbesondere bei den Sioux die Haplogruppe X nachgewiesen werden. Alle diese Haplogruppen werden auch bei Indianerskeletten aus der Zeit vor Kolumbus gefunden, so dass sie tatsächlich die Ureinwohner kenn-

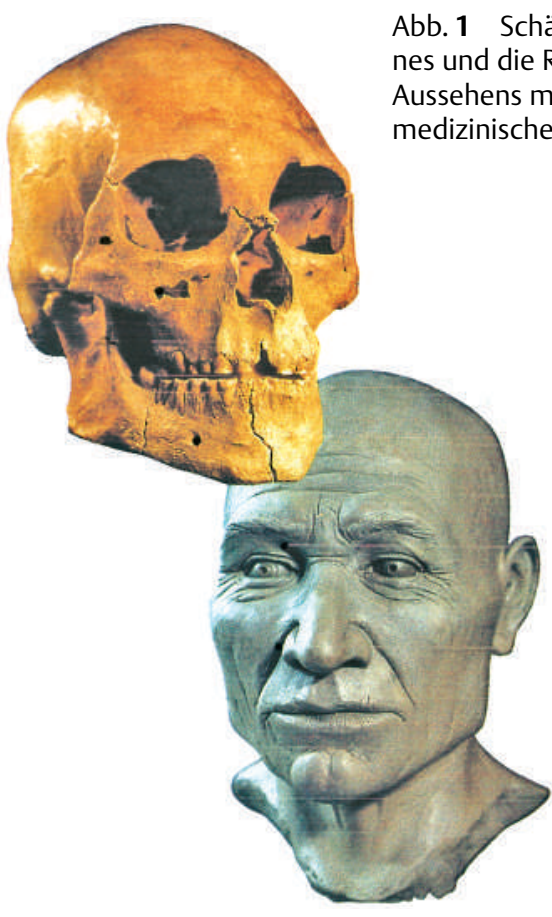

zeichnen. Die Haplogruppen A bis D treten ebenfalls in Asien auf und können somit die Herkunft der Indianer aus Asien belegen. Die Haplogruppe X dagegen ist im nördlichen Teil von Asien unbekannt und kommt in Europa vor. Vergleiche der gegenwärtigen europäischen und amerikanischen Haplogruppe $\mathrm{X}$ zeigen, dass die amerikanische Haplogruppe X vor mehr als 15000 Jahren von Europa nach Nordamerika gelangte [2].

Vermutlich waren die europäischen Solutréen-Menschen mit kleinen Kanus unterwegs. Sie fuhren entlang der Grenze von Meer und Eis über den Atlantik. Die Nächte verbrachten sie jeweils auf den Eisflächen und ernährten sich dort von der Jagd nach Seehunden oder fingen Fische. Mitten in der Eiszeit vollbrachten sie bei einem mörderischen Klima eine gewaltige Leistung. Nach Schätzungen stammt die Haplogruppe $\mathrm{X}$ der Indianer von etwa 10 Frauen ab. Es gibt in Nordamerika nur wenige Fundorte dieser Prä-ClovisKulturen (etwa Cactus Hill in Virginia). Ihre Pfeilspitzen sind ungefähr 5000 Jahre älter als die der Clovis-Menschen und entsprechen exakt den Arbeiten der europäischen Solutréen-Menschen.

\section{Wilkinger, Iren und Waliser}

Auch nach der Eiszeit machten sich immer wieder Menschen auf den Weg von Europa nach Amerika. Während der Antike war das Ziel allerdings weniger Nordamerika sondern hauptsächlich Südamerika und die Karibik. Im Gegensatz zu den Menschen des Solutréen handelte es sich nicht um Einwanderer, sondern mehr um Seefahrer, die vermutlich keine dauerhaften Siedlungen mit eigenständigen Bevölkerungsgruppen gründeten. Erst die Wikinger und mit großer Wahrscheinlichkeit auch die Iren und Waliser ließen sich dauerhaft in Nordamerika nieder [5,6]. Ihre Siedlungen gerieten später in Vergessenheit und alle Verbindungen zur Alten Welt rissen $a b$, so dass im Zeitalter der Entdeckungen niemand über „weiße“ Bevölkerungsgruppen in Amerika informiert war.

Neben wenigen archäologischen Funden wie beispielsweise Häuserreste und eine Schmiede nahe dem Ort L'Anse-aux-Meadows 
auf Neufundland bestätigen allein überlieferte Sagas, dass die Wikinger um das Jahr 1000 n. Chr. in Amerika waren und dort Siedlungen gegründet hatten. Der legendäre Wikinger Leif Erikson soll mit seinen Männern von Island aus in Amerika gelandet sein und das neuentdeckte Gebiet nach den wilden weinähnlichen Pflanzen „Vinland“ genannt haben. Sein berühmter Vater, Erik der Rote, soll vor ihm ebenfalls in Amerika gewesen sein. In der Grönland-Saga der Wikinger wird ein Land westlich von Grönland beschrieben, und die Saga des Thorfinn Karlsefni berichtet schließlich, dass er mit seinen Leuten in Amerika eine Dauersiedlung gründen wollte. Er soll um das Jahr 1005 mit 60 bis 160 Siedlern in Amerika gelandet sein. Wikingergebiete in Nordamerika sollen neben Vinland noch Markland und Helluland gewesen sein. Wie tief die Wikinger einst in den nordamerikanischen Kontinent verdrangen, ist unbekannt. Im US-Staat Maine wurde einmal eine Silbermünze des Wikingerkönigs Olaf Kyrri (11. Jahrhundert) gefunden. Umstritten ist der Stein von Kensington in Minnesota mit einer Runeninschrift. Er berichtet über Auseinandersetzungen zwischen Wikingern und Indianern im Jahre 1362, gilt allerdings für manche Fachleute als Fälschung.

Während des Hochmittelalters war es in Europa wärmer als heute, und in Grönland lebten etwa 3000 Wikinger überwiegend von der Viehzucht. Sie trieben Handel mit den amerikanischen Wikingersiedlungen und importierten hauptsächlich Holz. In einer grönländischen Wikingersiedlung wurde einmal eine typische Pfeilspitze der Indianer aus Labrador-Quarzit gefunden, die wahrscheinlich ein verwundeter Wikinger bei seiner Rückkehr im Körper trug. Der grönländische Bischof der christlichen Wikinger führte sogar ab 1120 den auf Dokumenten belegten Titel „Bischof von Grönland und Vinland“. Als sich am Ende des Mittelalters das Klima jedoch ständig verschlechterte, mussten die grönländischen Wikingersiedlungen aufgegeben werden, und viele Menschen kehrten wegen der Kälte nach Island zurück. Die grönländische Westsiedlung der Wikinger war beim Besuch einer bischöflichen Delegation um 1350 menschenleer. Opfer von Auseinandersetzungen mit den Eskimos wurden nicht gefunden. Die Bewohner waren vermutlich nach Amerika ausgewandert. Auf Neufundland lebte bis zu seinem Aussterben im Jahre 1829 das hellhäutige Volk der Beothuk, dessen Herkunft leider nie untersucht wurde. Wenig erforscht sind auch die Überlieferungen des Indianervolkes der Micmac, die von weißen Menschen in Neufundland berichten.

Als die Wikinger um 860 erstmals in Island landeten, lebten dort bereits irische Mönche. Die Seefahrerkünste der Iren sind zwar nicht so bekannt wie die der Wikinger, doch sie erreichten vermutlich mit ihren Rundbooten noch vor den Wikingern Amerika. Der irische Mönch Brendan soll um 531 mit 12 Begleitern in Amerika gewesen sein, das er nach seiner Rückkehr als das „Gelobte Land“ pries. Es wird vermutet, dass er an der Küste der heutigen amerikanischen Südstaaten angekommen war. Ob irische Familien später seinem Beispiel folgten und in Amerika Siedlungen gründeten, ist umstritten und lässt sich nicht sicher beweisen. Legenden berichteten allerdings von einem geheimnisvollen Hvitramannaland, auch Groß-Irland genannt, an der amerikanischen Ostküste, in dem weiße Menschen leben würden. Ein Kaufmann aus Island, dessen Schiff abgetrieben war, teilte um das Jahr 1000 mit, er sei in Hvittramannaland gestrandet und die Menschen hätten dort einen alten irischen Dialekt gesprochen.
Unter den Angehörigen des inzwischen ausgestorbenen Volkes der Tuscarora an der südlichen Ostküste der USA soll es zahlreiche europäisch aussehende Weiße gegeben haben. Nach einer Legende soll ein Waldläufer irischer Abstammung einmal von ihnen gefangen genommen worden sein. Er fürchtete um sein Leben und soll laut ein irisches Gebet gesprochen haben. Die „Indianer“ hätten ihn verstanden und zu ihrem Dorf gebracht, wo er freundlich bewirtet wurde.

Der walisische Prinz Madoc war der uneheliche Sohn von König Owain Gwynedds und musste nach dessen Tod das Erbe mit 19 Brüdern und Halbbrüdern teilen. Er beschloss auszuwandern, segelte westwärts und landete angeblich in Amerika. Später kehrte er wieder zurück, stellte eine kleine Flotte zusammen und warb mehr als 120 Siedler mit ihren Familien an. Sie segelten zusammen um 1170 erneut nach dem Westen und wurden nie mehr gesehen. Angeblich ließen sie sich zunächst im heutigen US-Staat Georgia nieder und erschlossen später vermutlich neue Siedlungsgebiete. In den US-Staaten Alabama, Tennessee und Kentucky gibt es uralte Mauerreste, die an Fundamente von Burgen erinnern und die sich völlig von Bauten der Indianer unterscheiden. In einer Legende der Cherokee wurde mitgeteilt, dass weiße Männer einst über das Meer gekommen waren und eine große Festung gebaut hätten. Madocs amerikanische Siedlungen waren lange allgemeines Wissensgut und wurden erst später vergessen. Der englische Staatsmann John Dee begründete mit der Landnahme von Madoc sogar den Anspruch von Königin Elisabeth I. auf Amerika [7,8].

\section{Die Vinland-Karte}

Es existiert sogar eine Darstellung von Amerika auf einer rätselhaften Landkarte, die auf das Jahr 1440 datiert wird. Die Vinland-Karte (Abb. 2) unterscheidet sich von den üblichen Landkarten ihrer Zeit in einem entscheidenden Punkt. Auf dem linken oberen Rand des Pergamentblattes, also im Westen, ist eine Insel mit dem Namen „Vinilanda“ abgebildet. Somit wäre die Karte, sollte sie echt sein, ein kartographischer Beleg für die europäische Entdeckung Amerikas vor Kolumbus. Einbuchtungen im Umriss der Insel wer-

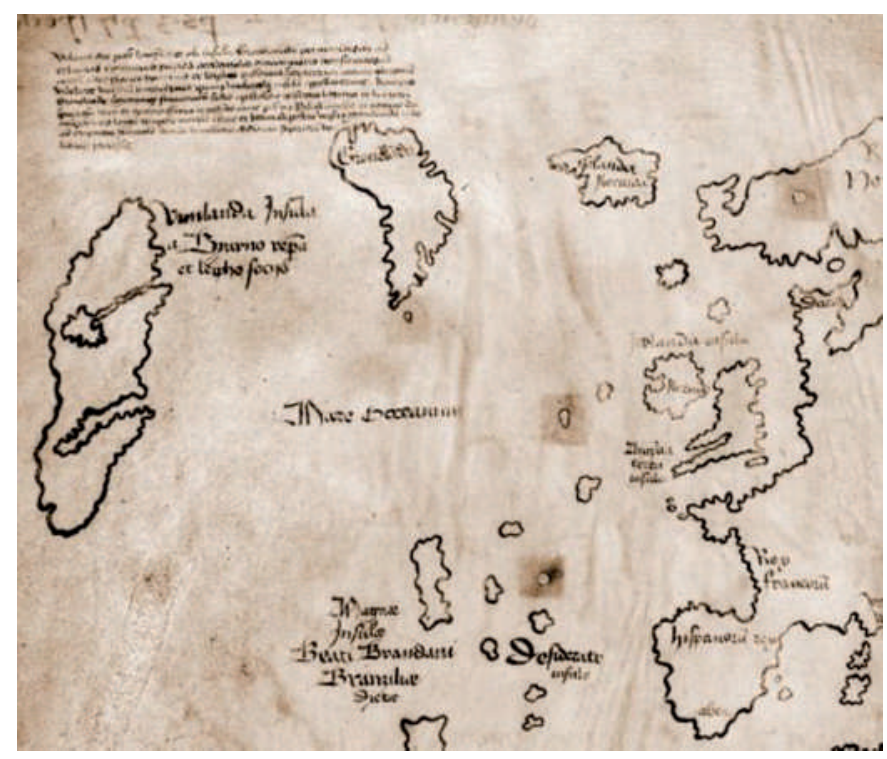

Abb. 2 Ausschnitt der Vinland-Karte. Links oben ist „Vinilanda“ dargestellt, der erste kartographische Hinweis auf Amerika, mehr als 50 Jahre vor Kolumbus (Yale University, New Haven, USA). 


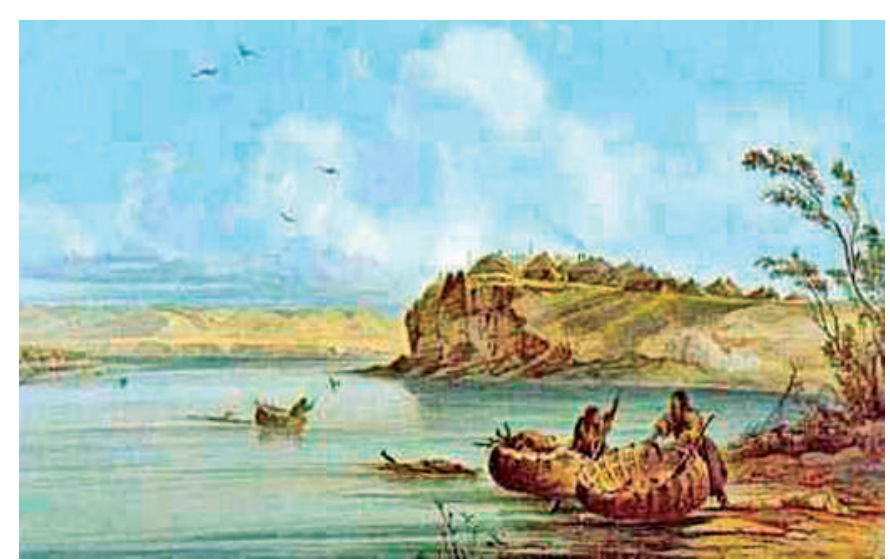

Abb. 3 Schiffe der Mandan. Sie sind wie keltische Rundschiffe konstruiert und unterscheiden sich völlig von den bei Indianern sonst üblichen Kanus.

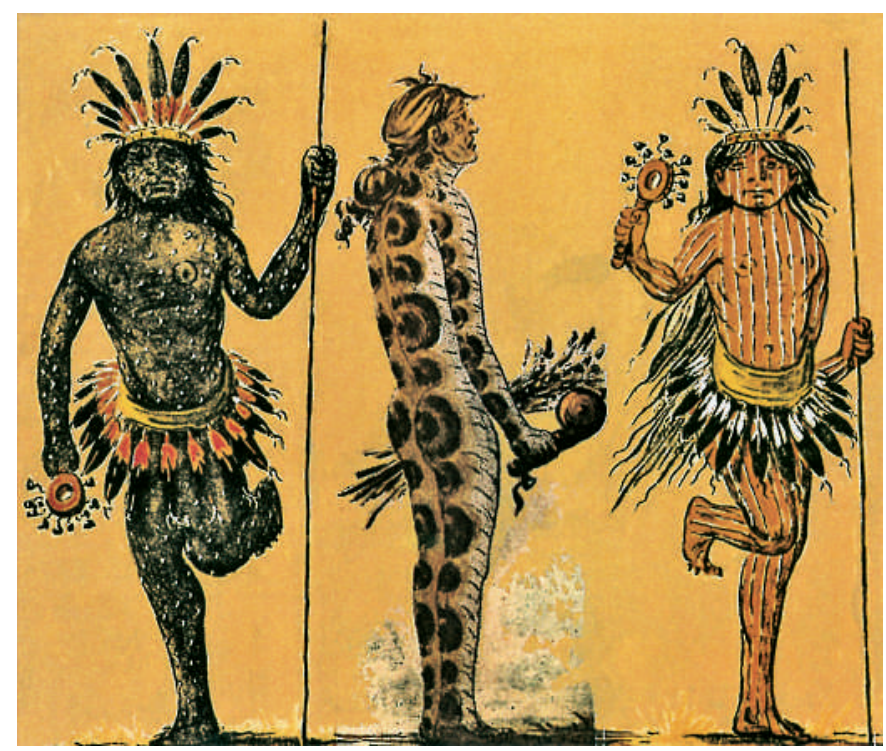

Abb 4 Tanzszene der Mandan nach einer Skizze von George Catlin. Der Tänzer in der Mitte besitzt blonde Haare. Mit einem solchen Tanz wurde vor der Jagd der Geist der Tiere beruhigt.

den als Hinweise auf die Hudson Bay und den St. Lorenz Strom gewertet. Die Karte gehört heute der Yale Universität. Materialanalysen der Karte belegen beides: Sie könnte sowohl echt als auch eine Fälschung sein. Das Pergament stammt zweifelsfrei aus der Zeit um 1440. An einigen Stellen der Zeichnung wurde jedoch Titanweiß gefunden, das es um 1440 noch nicht gab, so dass die Karte auch gefälscht sein könnte. Vielleicht bezieht sich das Titanweiß aber auch auf eine unsachgemäße Restaurierung, denn es kommt nicht an allen Stellen der Strichführung vor. Andere Forscher sind überzeugt, dass Vorstufen zum Titanweiß bereits den mittelalterlichen Alchimisten bekannt waren und durchaus um 1440 benutzt werden konnten [9].

\section{Das Volk der Mandan}

Nach der Gründung der Vereinigten Staaten drängten bald viele wagemutige Pioniere in das Innere des riesigen Landes. Sie wollten dort siedeln und schnell zu großem Reichtum gelangen. Obwohl es viele überraschende Entdeckungen gab, wurden die Lebensgrund-

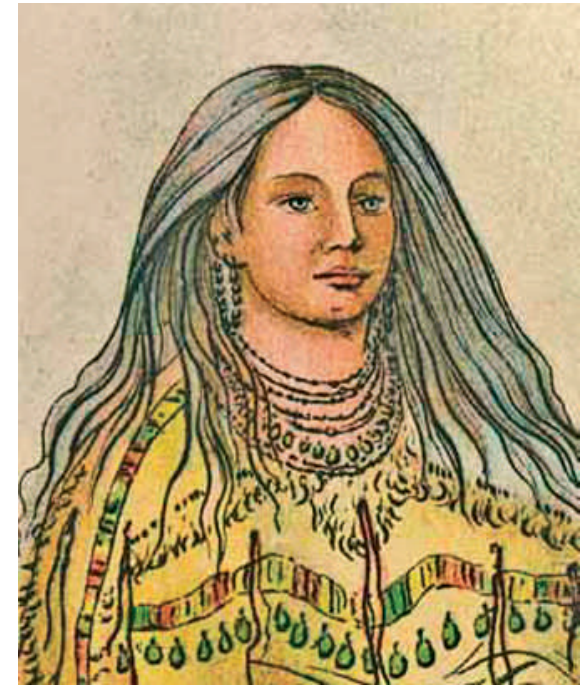

Abb 5 Sha-ko-ka, eine junge Frau der Mandan nach einer Skizze von George Catlin. Die Frau sieht nicht wie eine typische Indianerin aus, sondern erscheint wie eine Europäerin.

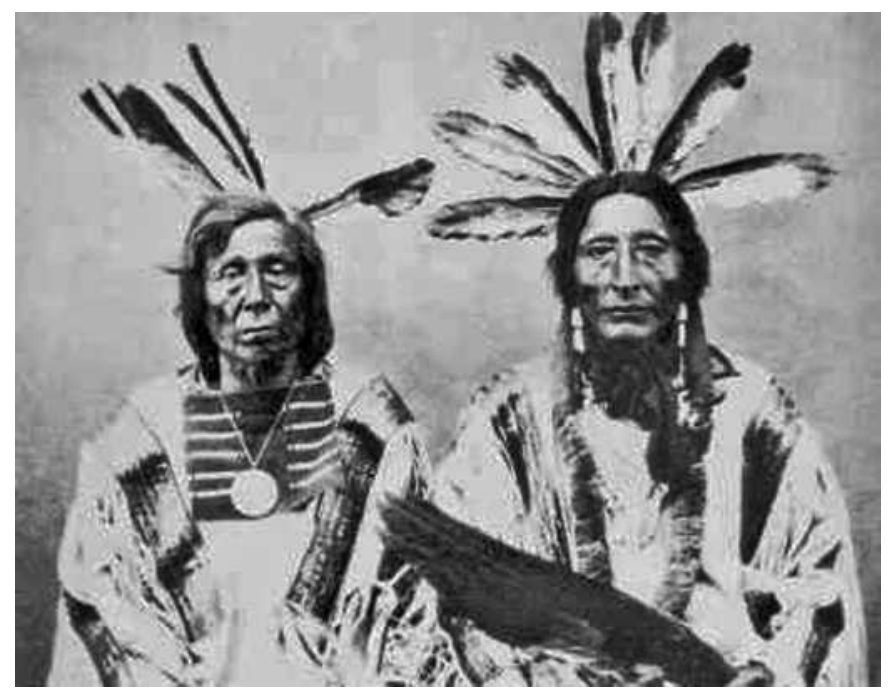

Abb. 6 Frühe Fotografie von zwei Mandan-Indianern, die die Pockenepidemie überlebt hatten. Der Mann links besitzt für einen Indianer sehr helle Haare (aus: Internet, Galerie der Mandan).

lagen und die kulturellen Leistungen der Indianer sinnlos zerstört. Frühe Waldläufer, Abenteurer und Pelzhändler berichteten immer wieder von Stämmen von „weißen Indianern“, die wie Europäer aussahen. Später konzentrierten sich die Meldungen auf das kleine Volk der Mandan-Indianer, das bei seiner Entdeckung im Einzugsgebiet des oberen Mississippi und Missouri lebte und aus nur rund 2000 Menschen bestand. Die Mandan waren aus einer unbekannten Gegend in dieses Gebiet eingewandert und dort sesshaft geworden. Sie betrieben Landwirtschaft sowie zeitlich begrenzt Jagd und lebten in wohlgeordneten Dörfern. Ihre Boote waren wie in Irland und Wales rund und nach dem Prinzip der typischen keltischen Rundboote, der Coracles, gefertigt (Abb. 3). In ihrer Sprache sollen sie keltische Worte verwendet haben.

Heute werden die Mandan zu den Sioux gezählt, aber sie sahen nicht wie echte Sioux-Indianer aus. Etwa 20 Prozent von ihnen hatten nach verschiedenen Quellen blaue Augen und eine so helle Haut wie Nordeuropäer. Ihre Haarfarbe war nicht tiefschwarz wie bei anderen Indianern, sondern mehr dunkel- und hellbraun oder 


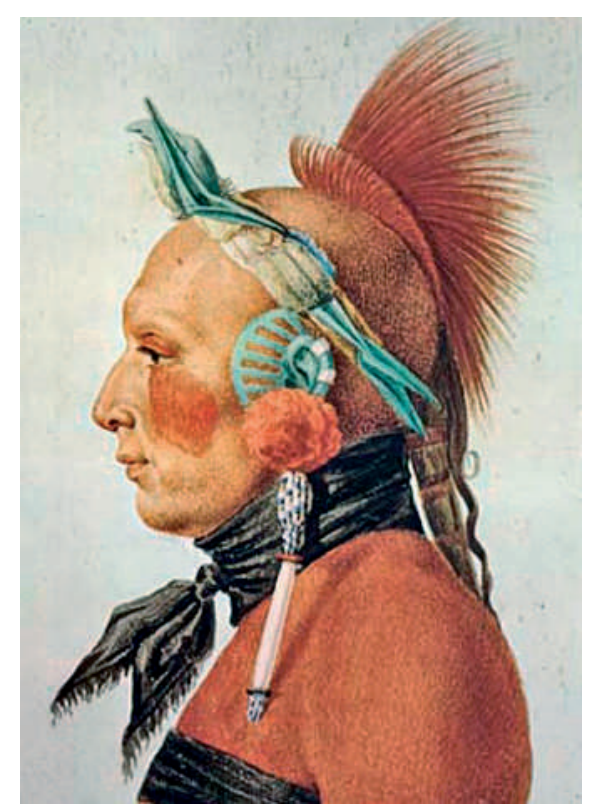

Abb. 7 Krieger der Osage bei einem Besuch bei Präsident Jefferson in Washington, Aquarell von Charles de Saint Memin. Das Profil des Mannes ist sehr stark europäisch.

sogar blond (Abb.4). Der amerikanische Maler George Catlin besuchte 1832 die Mandan und lebte einige Monate bei ihnen, um Zeichnungen und Skizzen anzufertigen. Ihn begeisterten die jungen Mandan-Frauen, die nach seiner Meinung wie europäische Schönheiten aussahen (Abb.5). Catlin war überzeugt, dass die Mandan von den walisischen Siedlern um Prinz Madoc abstammten. Diese Siedler wurden in Wales „Madawgwys“ genannt, was zu dem Namen „Mandan“ geführt haben könnte. In ihren Mythen verehrten die Mandan einen „weißen Mann“ als Urvater und kannten noch vor der Ankunft von christlichen Missionaren Relikte von Erzählungen aus der Bibel.

Bevor die großen Siedlungswellen das Gebiet der Mandan erreichten, waren sie bereits ausgestorben. Um 1838 hatte ein Dampfer aus St. Louis bei einem Dorf der Mandan angelegt und zwei Besatzungsmitglieder schleppten die Pocken ein. Die Seuche hatte verheerende Folgen. Noch nicht einmal 100 Mitglieder des Volkes der Mandan überlebten die Pockenerkrankungen (Abb.6). Dieser kümmerliche Rest vermischte sich später mit anderen Indianervölkern, so dass es außer wenigen Berichten wie etwa die des deutschen Prinzen und Forschungsreisenden Maximilian zu Wied und den Bildern von Catlin heute keine Informationen über das kleine Volk der Mandan gibt [10].

Indianer mit einem verblüffenden „europäischen Aussehen“ kamen nicht nur unter den Mandan vor (Abb. 7). Auch bei anderen Indianervölkern gab es Menschen, die nicht wie typische Indianer aussahen, nur war ihr Anteil an der Bevölkerung nicht so hoch wie bei den Mandan. Viele Mitglieder der Dakota hatten beispielsweise bei typisch indianischen Gesichtszügen eine weiße Haut.

\section{Literatur}

${ }^{1}$ Scarre C. The Human Past. London: Thames \& Hudson, 2005

${ }^{2}$ Olson S. Mapping Human History. Discovering the Past through our Genes. Boston, New York: Houghton Mifflin Co, 2002

${ }^{3}$ Herrmann J, Ullrich $\mathrm{H}$ (Hrsg). Menschwerdung. Millionen Jahre Menschheitsentwicklung. Berlin: Akademie Verlag, 1991

${ }^{4}$ Dillehay T. The Settlement of the Americas. New York: Basic Books, 2000

${ }^{5}$ Stein W (Hrsg). Kolumbus oder wer entdeckte Amerika? München: Hirmer, 1992

${ }^{6}$ Morison SE. The European Discovering of America - The Northern Voyages, A.D. 500 - 1600. Oxford: Oxford University Press, 1971

${ }^{7}$ Deacon R. Madoc and the Discovery of America. New York: George Braziller, Inc, 1966

${ }^{8}$ Hermann P. 7 vorbei und 8 verweht. Die Abenteuer der frühen Entdeckungen. Hamburg: Hofmann und Campe, 1952

${ }^{9}$ Reitz M. Auf der Fährte der Zeit. Mit naturwissenschaftlichen Methoden vergangene Rätsel entschlüsseln. Weinheim: Wiley-VCH, 2003

10 Schmitz H-P. Mandan/Madoc/Madawgwys. Die Suche nach den Wurzeln des Mandan-Volks. Magazin für Amerikanistik 2001; Heft 1

Dr. Manfred Reitz

Schillerstr. 7

99423 Weimar

mreitz@fli-leibniz.de

\section{Buchbesprechung}

\section{Antimykotika von $\mathbf{A}-\mathbf{Z}$}

H.-J. Tietz, W. Sterry

4. neu bearb. u. erw. Aufl. Stuttgart: Thieme, 2006. 140 S. Kart. 9,95 €. ISBN 3-13-137794-1

Pilzinfektionen betreffen den ganzen Menschen und alle Organe, besonders aber die Haut. Demnach sind alle Fachkollegen mit Mykosen konfrontiert, aber besonders häufig wir Dermatologen. Dies zumal wir auch Venerologen sind, Kinder, Jugendliche und Senioren behandeln und über Organmykosen Bescheid wissen müssen. Aus der Berliner Charité entstand 1999 ein handliches Büchlein zur Anwendung und Pharmakologie der Mykosen auf einen Blick. Es enthielt Kapitel über Einteilung der Antimykotika, deren Beschreibung und zur Therapie von Problemmykosen. Es war hilfreich und erfolgreich, so dass jetzt die 4. Auflage in 7 Jahren vorliegt. Sie stammt wieder aus der Charité und nun auch aus dem neuen und dynamischen Institut für Pilzkrankheiten in Berlin. Die Einteilung ist geblieben, einige Verluste in der Palette der Wirkstoffe und Anwendungsweisen werden offen gelegt und Neuerungen werden vorgestellt. Besonders wird die systemische Aspergillose als Problem bewusst gemacht. Es handelt sich um ein wirklich brauchbares, knapp gefasstes kleines Werk von 139 Seiten; ein echtes Taschenbuch von unschätzbarem Wert!

E. G. Jung, Heidelberg 